\title{
HARMONIC ANALYSIS ON SEMISIMPLE LIE GROUPS
}

\section{BY HARISH-CHANDRA}

1. Introduction. Let $G$ be a locally compact group which we assume to be separable and unimodular. Let $d x$ denote the Haar measure on $G$. If $\pi$ is a unitary representation of $G$ on a Hilbert space $\mathfrak{S}$ and $f \in L_{1}(G)$, we write

$$
\pi(f)=\int_{G} f(x) \pi(x) d x .
$$

Then $\pi(f)$ is a bounded operator on $\mathfrak{S}$ and

$$
\pi(f * g)=\pi(f) \pi(g)\left(f, g \in L_{1}(G)\right),
$$

where $f * g$ denotes the convolution of $f$ and $g$.

Let $A$ be a bounded linear operator on $\mathfrak{S}$. We say that $A$ is of the trace class if the series

$$
\sum_{i}\left|\left(\psi_{i}, A \psi_{i}\right)\right|
$$

converges for every orthonormal base $\left\{\psi_{i}\right\}_{i \in J}$ of $\mathfrak{S}$. Moreover if this is so, we define

$$
\operatorname{tr} A=\sum_{i}\left(\psi_{i}, A \psi_{i}\right)
$$

Then $\operatorname{tr} A$ is actually independent of the choice of this base.

Let $V_{\pi}$ denote the set of all $f \in L_{1}(G)$ such that $\pi(f)$ is of the trace class. Then $V_{\pi}$ is a linear subspace of $L_{1}(G)$. Put

$$
\Theta_{\pi}(f)=\operatorname{tr} \pi(f) \quad\left(f \in V_{\pi}\right) .
$$

Then $\Theta_{\pi}$ is a linear function on $V_{\pi}$ which we may call the character of $\pi$. Of course this concept would be useful only when the space $V_{\pi}$ is fairly large.

Let $\mathcal{E}(G)$ denote the set of all equivalence classes of irreducible unitary representations of $G$. It is easy to see that for any representation $\pi, V_{\pi}$ and $\Theta_{\pi}$ depend only on the class $\omega$ of $\pi$. Hence we may de-

A Colloquium Lecture delivered before the 74th Summer meeting of the Society at Eugene, Oregon, on August 26, 1969; received by the editors October 29, 1969.

AMS Subject Classifications. Primary 2265, 2260.

Key Words and Phrases. Characters, invariant eigendistributions, parabolic subgroups, Schwartz space, cusp forms, discrete series, Fourier transform of a Schwartz function, Plancherel formula, $p$-adic groups. 
note them by $V_{\omega}$ and $\Theta_{\omega}$ respectively. Put

$$
V_{0}=\bigcap_{\omega \in \mathcal{E}(G)} V_{\omega}
$$

For any function $f \in L_{1}(G)$ and $x \in G$, define the function $f^{x}$ by $f^{x}(y)=f\left(x^{-1} y x\right)$. It is clear that $f^{x} \in V_{0}$ whenever $f \in V_{0}$ and therefore the group $G$ operates on $V_{0}$. Now, in order to describe the main problem of harmonic analysis, we have to fix a certain topological vector space $V$ satisfying the following conditions: ${ }^{1}$

(1) $V \subset V_{0} \cap C(G)$, where $C(G)$ is the space of all complex-valued continuous functions on $G$.

(2) $V$ is stable under $G$ and every $x \in G$ defines a continuous endomorphism of $V$.

(3) For any $\omega \in \mathcal{E}(G)$, the restriction of $\Theta_{\omega}$ on $V$ is a continuous linear function on $V$, which determines $\omega$ uniquely.

For any $f \in V$, define $\hat{f}(\omega)=\Theta_{\omega}(\hat{f})(\omega \in \mathcal{E}(G))$. Then $\hat{f}$ is a complexvalued function on $\varepsilon(G)$, which may be regarded as the Fourier transform of $f$. Let us agree to call a continuous linear function on $V$, a distribution. For any distribution $T$ and $x \in G$, define the distribution $T^{x}$ by $T^{x}(f)=T\left(f^{x^{-1}}\right)(f \in V)$. We say that $T$ is invariant if $T^{x}=T$ for all $x \in G$.

The central question in harmonic analysis may now be formulated as follows. Given an invariant distribution $T$ on $G$, how to express it as a "linear combination" of the characters $\Theta_{\omega}(\omega \in \mathcal{E}(G))$ ? In fact, to every such $T$, we would like to associate a "distribution" $\hat{T}$ on $\mathcal{E}(G)$ in such a way that $T(f)=\hat{T}(\hat{f})$ for all $f \in V$. (Here by a "distribution" on $\varepsilon(G)$, we mean some sort of linear functional on a suitable space of functions on $\varepsilon(G)$.) If $\delta$ is the Dirac measure, so that $\delta(f)=f(1)(f \in V)$, the determination of $\hat{\delta}$ is just the problem of the explicit Plancherel formula for $G$.

For an arbitrary $G$, our understanding of $\varepsilon(G)$ is still very rudimentary (see [5]) and therefore no serious attempt to attack the above problem can yet be contemplated. However the situation for real semisimple (or reductive) Lie groups seems rather encouraging $[4(a),(d)]$ and there is reason to hope that some substantial results on reductive $\mathfrak{p}$-adic groups may be attainable within a few years. Therefore I shall limit myself to these two cases in these lectures. The other extreme case, when $G$ is a nilpotent or solvable Lie group, has been studied extensively by Dixmier, Kirillov, Moore, and Pukánszky (see [6], [7]).

${ }^{1}$ We do not claim here that such a space $V$ necessarily exists. Of course the crucial condition is (3). 
Our entire approach to harmonic analysis on reductive groups is based on the philosophy of cusp forms (see [2] and $[4(\mathrm{c}),(\mathrm{d})]$ ) and the success of this method depends on a good knowledge of the discrete series (see \$3) for such groups. In the case of real groups the characters of the discrete series are all known (see Theorem 8) and therefore the theory works reasonably well. But for $\mathfrak{p}$-adic groups our understanding of the supercuspidal representations ( $\$ 14)$ is still very poor. Nevertheless the resemblance between the two cases is so striking that one cannot give up the hope that the same philosophy would eventually work in both cases.

2. Characters in the real case. Suppose $G$ is a real, connected, semisimple Lie group with finite center. Fix a maximal compact

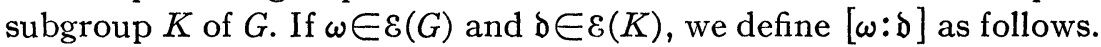
Fix a representation $\pi \in \omega$ and let $\pi_{K}$ denote the restriction of $\pi$ on $K$. Then $[\omega: \delta]$ is the multiplicity of $\delta$ in $\pi_{K}$. Since $K$ is compact, every irreducible representation of $K$ is finite-dimensional. We denote by $d(\delta)$ the degree of a representation in the class $\delta$.

THEOREM 1. There exists an integer $N \geqq 1$ such that

$$
[\omega: \mathfrak{D}] \leqq N d(\mathfrak{D})
$$

for all $\omega \in \mathcal{E}(G)$ and $\mathfrak{b} \in \mathcal{E}(K)$.

Let $C_{c}^{\infty}(G)$ denote the space of all complex-valued $C^{\infty}$ functions on $G$ with compact support, taken with its usual topology [9]. The following result is an easy consequence of Theorem 1.

THEOREM 2. Let $\pi$ be an irreducible unitary representation of $G$. Then for any $f \in C_{c}^{\infty}(G), \pi(f)$ is of the trace class. Put

$$
\Theta_{\pi}(f)=\operatorname{tr} \pi(f) \quad\left(f \in C_{c}^{\infty}(G)\right) .
$$

Then $\Theta_{\pi}$ is a distribution on $G$ in the sense of Schwartz [9].

As we have seen in $\S 1, \Theta_{\pi}$ depends only on the class $\omega$ of $\pi$. Hence we may denote it by $\Theta_{\omega}$.

TheOREM 3. Let $\omega_{1}, \omega_{2}$ be two elements in $\mathcal{E}(G)$. Then $\Theta_{\omega_{1}}=\Theta_{\omega_{2}}$ if and only if $\omega_{1}=\omega_{2}$.

This shows that if we take $V=C_{c}^{\infty}(G)$, then all the conditions of $\$ 1$ are fulfilled.

Let $u$ be a differential operator on $G$. Then its adjoint $u^{*}$ is the differential operator given by the relation

$$
\int_{G} u^{*} f \cdot g d x=\int_{G} f \cdot u g d x \quad\left(f, g \in C_{c}^{\infty}(G)\right) .
$$


If $T$ is a distribution on $G$, the mapping

$$
f \rightarrow T\left(u^{*} f\right) \quad\left(f \in C_{c}^{\infty}(G)\right),
$$

is also a distribution which we denote by $u T$. Let $F$ be a locally summable function on $G$. Put

$$
T_{F}(f)=\int F f d x \quad\left(f \in C_{c}^{\infty}(G)\right) .
$$

Then $T_{F}$ is a distribution on $G$. We say that $T=F$ if $T=T_{F}$.

Let 3 be the algebra of all differential operators on $G$ which commute with both left and right translations of $G$. Then $Z$ is abelian. A distribution $T$ is called $B$-finite if the space of all distributions of the form $z T(z \in \mathbb{Z})$ has finite dimension. Moreover $T$ is said to be an eigendistribution of 3 , if this space has dimension $\leqq 1$. Finally we say that $T$ is $K$-finite, if the left and right translates of $T$ by elements of $K$, span a finite-dimensional space.

Fix an indeterminate $t$ and let $l=\operatorname{rank} G$. For any $x \in G$, let $D(x)$ denote the coefficient of $t^{l}$ in the polynomial $\operatorname{det}(t+1-\operatorname{Ad}(x))$. Then $D$ is an analytic function on $G$ which is not identically zero. A point $x \in G$ is called regular if $D(x) \neq 0$. Let $G^{\prime}$ denote the set of all regular points of $G$. Then $G^{\prime}$ is an open dense subset of $G$ whose complement is of measure zero.

THEOREM 4. Let $\Theta$ be an invariant and B-finite distribution on $G$. Then there exists an analytic function $F$ on $G^{\prime}$ such that $F$ is locally summable on $G$ and $\Theta=F$.

Fix $\omega \in \mathcal{E}(G)$ and let $\Theta_{\omega}$ denote, as before, the character of $\omega$. It is easy to see that $\Theta_{\omega}$ is an invariant eigendistribution of 3 . Therefore it follows from Theorem 4 that $\Theta_{\omega}$ is a function.

3. The discrete series. We return to the assumptions of $\$ 1$. Let $\pi$ be an irreducible unitary representation of $G$ on a Hilbert space $\mathfrak{S}$. We say that $\pi$ is of type $L_{p}(p \geqq 1)$, if there exist nonzero elements $\phi, \psi \in \mathfrak{S}$ such that

$$
\int_{a}|(\phi, \pi(x) \psi)|^{p} d x<\infty .
$$

In case $\pi$ is of type $L_{2}$ (or, as we sometimes say, square-integrable), there exists a positive number $d(\pi)$ such that ${ }^{2}$

${ }^{2}$ conj $c(c \in C)$ denotes the complex conjugate of $c$. 


$$
\int_{G}\left(\phi_{1}, \pi(x) \psi_{1}\right) \cdot \operatorname{conj}\left(\phi_{2}, \pi(x) \psi_{2}\right) d x=d(\pi)^{-1}\left(\phi_{1}, \phi_{2}\right) \cdot \operatorname{conj}\left(\psi_{1}, \psi_{2}\right)
$$

for all $\phi_{i}, \psi_{i} \in \mathfrak{S}(i=1,2)$ (see [3] and [5, p. 640]). These are called the Schur orthogonality relations for $\pi$ and $d(\pi)$ the formal degree of $\pi$. Let $\varepsilon_{p}(G)$ denote the set of all classes $\omega \in \mathcal{E}(G)$ which contain representations of type $L_{p}$. If $\omega \in \mathcal{E}_{2}(G)$, we put $d(\omega)=d(\pi)$ for $\pi \in \omega$. The set $\mathcal{E}_{2}(G)$ is called the discrete series for $G$.

Now suppose $K$ is an open and compact subgroup of $G$. Normalize $d x$ in such a way that the total measure of $K$ is 1 . For any $\omega \in \mathcal{E}(G)$ and $\downarrow \in \varepsilon(K)$, let $[\omega: \delta]$ denote, as before (see $\S 2$ ), the multiplicity of $\delta$ in $\omega$ and $d(\delta)$ the degree of $\delta$.

Theorem 5. Fix $b \in \mathcal{E}(K)$. Then

$$
d(\delta) \geqq \sum_{\omega \in \varepsilon_{2}(G)} d(\omega)[\omega: \delta]
$$

This is a simple consequence of the Schur orthogonality relations.

Corollary. Fix $\omega \in \mathcal{E}_{2}(G)$. Then

$$
[\omega: \S] \leqq d(\omega)^{-1} d(\S)
$$

for all $b \in \mathcal{E}(K)$.

4. Characters of the discrete series (real case). Define $G$ as in $\$ 2$.

Theorem 6. $\varepsilon_{2}(G) \neq \varnothing$ if and only if $G$ has a compact Cartan subgroup.

Let us now assume that $G$ has a compact Cartan subgroup $B$. Then any two such Cartan subgroups are conjugate in $G$. Let $g$ be the Lie algebra of $G$ and $\mathfrak{g}_{c}$ its complexification. Let $G_{c}$ be the simply connected complex-analytic group corresponding to $\mathfrak{g}_{c}$. For simplicity we assume that $G$ is the real analytic subgroup of $G_{c}$ corresponding to $\mathrm{g}$.

Let $\mathfrak{b}$ be the Lie algebra of $B$ and $\mathfrak{b}^{*}$ the dual of $\mathfrak{b}$. Fix some order on the real vector space $(-1)^{1 / 2} \mathfrak{b}^{*}$. Let $W=W(\mathfrak{g} / \mathfrak{b})$ denote the Weyl group of ${ }^{3}\left(\mathfrak{g}_{c}, \mathfrak{b}_{c}\right)$ and $B^{*}$ the character group of $B$. Then $W$ operates on $\mathfrak{b}$ and $B$ and therefore by duality, it also operates on $\mathfrak{b}^{*}$ and $B^{*}$. Let $\left\langle b^{*}, b\right\rangle\left(b^{*} \in B^{*}, b \in B\right)$ denote the value of the character $b^{*}$ at $b$. Then we have the relations

${ }^{8}$ For any finite-dimensional vector space $V$ over $R$, we denote by $V^{*}$ its dual and by $V_{0}$ its complexification. Moreover $V_{0}^{*}=\left(V^{*}\right)_{0}$. 


$$
(\exp H)^{s}=\exp (s H), \quad\left\langle b^{*_{s}}, b\right\rangle=\left\langle b^{*}, b^{s^{-1}}\right\rangle
$$

for $H \in \mathfrak{b}$ and $s \in W$.

Put

$$
\varpi=\prod_{\alpha \in P} H_{\alpha}
$$

where $P$ is the set of all positive roots of $(\mathfrak{g}, \mathfrak{b})$ and $H_{\alpha}$ the element in $\mathfrak{b}_{c}$, which is dual to $\alpha$ under the Killing form so that

$$
\alpha(H)=\operatorname{tr}\left(\operatorname{ad} H \cdot \operatorname{ad} H_{\alpha}\right) \quad(H \in \mathfrak{b}) .
$$

Then $\varpi$ is a polynomial function on $\mathfrak{b}_{c}^{*}$ and $\varpi^{s}=\epsilon(s) \varpi(s \in W)$ where $\epsilon(s)=1$ or -1 . Moreover there exists an analytic function $\Delta$ on $B$ such that

$$
\Delta(\exp H)=\prod_{\alpha \in P}\left(e^{\alpha(H) / 2}-e^{-\alpha(H) / 2}\right) \quad(H \in \mathfrak{b})
$$

For any $b^{*} \in B^{*}$, let $\log b^{*}$ denote the element $\lambda \in(-1)^{1 / 2} \mathfrak{b} *$ such that

$$
\left\langle b^{*}, \exp H\right\rangle=e^{\lambda(H)} \quad(H \in \mathfrak{b})
$$

and put

$$
\varpi\left(b^{*}\right)=\varpi\left(\log b^{*}\right) \quad\left(b^{*} \in B^{*}\right) .
$$

We say that $b^{*}$ is regular if $\varpi\left(b^{*}\right) \neq 0$.

Let $\widetilde{B}$ be the normalizer of $B$ in $G$. Then $W(G / B)=\widetilde{B} / B$ may be regarded as a subgroup of $W$.

TheOREM 7. Fix $b^{*} \in B^{*}$. Then there exists an invariant eigendistribution $\Theta$ of $\$$ on $G$ such that 4

(1) $\sup _{x \in G^{\prime}}|D(x)|^{1 / 2}|\Theta(x)|<\infty$,

(2) $\Theta=\Delta^{-1} \sum_{s \in W(G / B)} \epsilon(s) b^{*_{s}}$ pointwise on $B \cap G^{\prime}$.

Moreover $\Theta$ is unique if $\varpi\left(b^{*}\right) \neq 0$.

Let $B^{* \prime}$ be the set of all regular elements in $B^{*}$ and for any $b^{*} \in B^{* \prime}$, let $\Theta_{b} *$ denote the corresponding distribution of Theorem 7. Put $\epsilon\left(b^{*}\right)=\operatorname{sign} \varpi\left(b^{*}\right)$ and $q=\frac{1}{2} \operatorname{dim} G / K$.

THEOREM 8. Given $b^{*} \in B^{* \prime}$, there exists a unique class $\omega\left(b^{*}\right) \in \mathcal{E}_{2}(G)$ such that

$$
(-1)^{q} \epsilon\left(b^{*}\right) \Theta_{b^{*}}
$$

is the character of $\omega\left(b^{*}\right)$. The mapping $b^{*} \rightarrow \omega\left(b^{*}\right)$ from $B^{* \prime}$ to $\varepsilon_{2}(G)$ is

\footnotetext{
- Here we have to keep in mind Theorem 4.
} 
surjective. Moreover $\omega\left(b_{1}^{*}\right)=\omega\left(b_{2}^{*}\right)$ if and only if $b_{1}^{*}, b_{2}^{*}$ are conjugate under $W(G / B)$.

Let $\sigma$ be an automorphism of $G$ such that $\sigma B=B$. Then $\sigma$ also operates on $B^{*}$ by the relation

$$
\left\langle\sigma b^{*}, b\right\rangle=\left\langle b^{*}, \sigma^{-1} b\right\rangle \quad\left(b^{*} \in B^{*}, b \in B\right) .
$$

Define $\epsilon(\sigma)= \pm 1$ by the rule $\omega^{\sigma}=\epsilon(\sigma) \varpi$. Also if $T$ is a distribution on $G$, we define the distribution $T^{\sigma}$ by

$$
T^{\sigma}(f)=T\left(f^{\prime}\right) \quad\left(f \in C_{c}^{\infty}(G)\right),
$$

where $f^{\prime}(x)=f(\sigma x)(x \in G)$.

LEMmA 1. Let $\sigma$ be an automorphism of $G$ such that $\sigma B=B$. Then

$$
\left(\Theta_{b^{*}}\right)^{\sigma}=\epsilon(\sigma) \Theta_{\sigma b^{*}} \quad\left(b^{*} \in B^{* \prime}\right) .
$$

Actually it is possible to define $\Theta_{b^{*}}$ for all $b^{*} \in B^{*}$ in such a way that both Theorem 7 and Lemma 1 hold for all $\sigma$.

5. Parabolic subgroups. Let $G$ be as in $\S 2$. By a parabolic subgroup (psgp) $P$ of $G$, we mean a closed subgroup of $G$ with the following two properties.

(1) If $\mathfrak{p}$ is the Lie algebra of $P$, then $\mathfrak{p}_{c}$ contains a Borel subalgebra (i.e. a maximal solvable subalgebra) of $g_{c}$.

(2) $P$ is the normalizer of $\mathfrak{p}$ in $G$.

By the radical $N$ of $P$, we mean the maximal normal subgroup of $P$ such that $\operatorname{Ad}(n)$ is unipotent for every $n \in N$. An abelian subgroup $A$ of $G$ is said to split if, for every $a \in A, \operatorname{Ad}(a)$ can be diagonalized over $R$. Fix $K$ as in $\$ 2$ and let $\theta$ denote the Cartan involution of $G$ corresponding to $K$. Put $M_{1}=P \cap \theta(P)$ and let $A$ be a maximal connected split abelian subgroup lying in the center of $M_{1}$. Then $A$ is unique and $M_{1}$ is the centralizer of $A$ in $G$. Let $X\left(M_{1}\right)$ denote the group of all continuous homomorphisms of $M_{1}$ into the multiplicative group $R^{\times}$ of real numbers. Put

$$
M=\bigcap_{\chi \in X\left(M_{1}\right)} \operatorname{ker}|\chi|,
$$

where $|\chi|(m)=|\chi(m)|\left(m \in M_{1}\right)$. Then $M_{1}=M A, P=M A N$ and the corresponding mapping of $M \times A \times N$ into $P$ is a diffeomorphism. We call this the Langlands decomposition of $P$ and $A$ the split component of $P$. Let $\mathfrak{a}$ be the Lie algebra of $A$. Then the exponential mapping defines a bijection of $\mathfrak{a}$ on $A$ and we denote its inverse by $\log$. By the parabolic rank of $P$ we mean the dimension of $A$ and denote it by prk $P$. 
The group $M$ is, in general, neither connected nor semisimple. However it is always reductive. We say that the psgp $P$ is cuspidal if $M$ has a compact Cartan subgroup.

Let $\mathfrak{h}$ be a Cartan subalgebra of $\mathfrak{g}$. We denote by $\mathfrak{h}_{R}$ the set of all points in $\mathfrak{b}$ where every root of $(\mathfrak{g}, \mathfrak{h})$ takes a real value.

Lemma 2. A psgp $P=M A N$ is cuspidal if and only if there exists a $\theta$-stable Cartan subalgebra $\mathfrak{h}$ of $\mathfrak{g}$ such that $\mathfrak{a}=\mathfrak{h}_{R}$.

Let $P=M A N$ be a prgp of $G$. Then $G=K P$. Hence every element $x \in G$ can be written in the form $x=k \operatorname{man}(k \in K, m \in M, a \in A, n \in N)$. The element $a$ is uniquely determined and we put $H_{P}(x)=\log a$. Let $\mathfrak{a}^{*}$ be the dual of $\mathfrak{a}$ and $\mathfrak{n}$ the Lie algebra of $N$. Then we define $\rho_{P} \in \mathfrak{a}^{*}$ by

$$
\rho_{P}(H)=\frac{1}{2} \operatorname{tr}(\operatorname{ad} H)_{\mathfrak{n}} \quad(H \in \mathfrak{a}),
$$

where $(\operatorname{ad} H)_{\mathfrak{n}}$ denotes the restriction of ad $H$ on $\mathfrak{n}$. By a root of $(P, A)$ (or $P$ ) we mean an element $\alpha \in \mathfrak{a}^{*}$ with the following property. There should exist an element $X \neq 0$ in $\mathfrak{n}$ such that $[H, X]=\alpha(H) X$ for all $H \in \mathfrak{a}$. Let $\mathfrak{a}^{+}$be the set of all $H \in \mathfrak{a}$ such that $\alpha(H) \geqq 0$ for every root $\alpha$ of $(P, A)$. We put $A^{+}=\exp \mathfrak{a}^{+}$.

Two psgps $P_{1}, P_{2}$ are said to be associated if their split components $A_{1}, A_{2}$ are conjugate under $G$ (or equivalently under $K$ ).

A psgp $P$ is called minimal if it is minimal among all psgps of $G$. The following three conditions are mutually equivalent.

(1) $P=M A N$ is a minimal psgp.

(2) $M \subset K$.

(3) prk $P=\operatorname{rank} G / K$.

Any two minimal psgps are conjugate under $K$.

6. The functions $\Xi$ and $\sigma$. Fix a minimal psgp $P=M A N$ of $G$. Then $G=K A N$ and this is an Iwasawa decomposition of $G$. Put

$$
\Xi(x)=\int_{\mathbf{K}} e^{-\rho(H(x k))} d k \quad(x \in G)
$$

where $H(x)=H_{P}(x), \rho=\rho_{P}$ and the Haar measure $d k$ on $K$ is so normalized that the total measure of $K$ is 1 .

Define a norm on $g$ by setting

$$
\|X\|^{2}=-\operatorname{tr}(\operatorname{ad} X \operatorname{ad} \theta(X)) \quad(X \in \mathfrak{g}) .
$$

Then since $G=K A K$, there exists a unique function $\sigma$ on $G$ such that

(1) $\sigma\left(k_{1} x k_{2}\right)=\sigma(x) \quad\left(k_{1}, k_{2} \in K, x \in G\right)$,

(2) $\sigma(\exp H)=\|H\| \quad(H \in \mathfrak{a})$. 
The functions $\Xi$ and $\sigma$ are both independent of the choice of $P$ and they have the following remarkable properties.

(1) $\int_{K} \Xi(x k y) d k=\Xi(x) \Xi(y) \quad(x, y \in G)$.

(2) There exist numbers $c, d \geqq 0$ such that

$$
1 \leqq \Xi(a) e^{\rho(\log a)} \leqq c(1+\sigma(a))^{d} \quad\left(a \in A^{+}\right) .
$$

(3) We can choose $r_{0} \geqq 0$ such that

$$
\int_{G} \Xi(x)^{2}(1+\sigma(x))^{-r_{0}} d x<\infty .
$$

(4) There exists a number $r \geqq 0$ such that ${ }^{5}$

$$
\int_{\sigma}|D(x)|^{-1 / 2} \Xi(x)(1+\sigma(x))^{-r} d x<\infty .
$$

Let $\mathfrak{q}$ be any linear subspace of $\mathfrak{g}$. Then we denote by $d \mathfrak{q}$ the Euclidean measure on $q$ corresponding to the above Euclidean norm on $\mathrm{g}$. It is convenient to normalize the Haar measure $d x$ on $G$ as follows. Let $d a$ and $d n$ denote the Haar measures on $A$ and $N$ which corespond to $d \mathfrak{a}$ and $d \mathfrak{n}$ respectively under the exponential mapping. Then if $x=$ kan $(k \in K, a \in A, n \in N)$,

$$
d x=e^{2 \rho(\log a)} d k d a d n
$$

where $d k$ is the normalized Haar measure of $K$. This normalization of $d x$ is independent of the choice of the minimal psgp $P$.

7. The Schwartz space and the cusp forms. Let $D$ be the algebra of all differential operators on $G$. Let $D_{l}$ and $D_{r}$ denote the subalgebras consisting of those $D \in D$ which commute with the left and right translations of $G$ respectively. Let $D_{0}$ be the subalgebra of $D$ generated by $D_{l} \cup D_{r}$.

For $f \in C^{\infty}(G), D \in D_{0}$ and $r \geqq 0$, put

$$
\nu_{D, r}(f)=\sup _{G}|D f|(1+\sigma)^{r} \Xi^{-1} .
$$

Let $\mathbb{e}(G)$ denote the space of all $f \in C^{\infty}(G)$ such that $\nu_{D, r}(f)<\infty$ for all $D \in \mathscr{D}_{0}$ and $r \geqq 0$. We topologize $\mathfrak{C}(G)$ by means of the set of seminorms $\nu_{D, r}\left(D \in D_{0}, r \geqq 0\right)$. Then $\mathfrak{C}(G)$ is a Hausdorff, locally convex and complete space and we call it the Schwartz space of $G$. We list here some of the properties of this space.

(1) The inclusion mapping of $C_{c}^{\infty}(G)$ into $\mathbb{e}(G)$ is continuous and the image is dense in $\mathfrak{e}(G)$.

${ }^{5}$ Here $D$ has the same meaning as in $\$ 2$. 
(2) $\mathcal{e}(G) \subset L_{2}(G)$.

(3) $\mathfrak{e}(G)$ is closed under convolution and the mapping $(f, g)$ $\rightarrow f * g(f, g \in \mathbb{C}(G))$ is continuous.

For $f \in \mathfrak{e}(G)$ and $P=M A N$ a psgp of $G$, define

$$
f^{P}(x)=\int_{N} f(x n) d n \quad(x \in G)
$$

where $d n$ is the Haar measure on $N$. (This integral always exists.) We say that $f$ is a cusp form if $f^{P}=0$ for every psgp $P \neq G$. Let ${ }^{\circ} \mathrm{e}(G)$ denote the space of all cusp forms on $G$. Then ${ }^{\circ} \mathfrak{C}(G)$ is a closed subspace of $\mathfrak{e}(G)$.

Theorem 9. Every $K$-finite and 3-finite function in $L_{2}(G)$ is a cusp form. Conversely $K$-finite eigenfunctions of 3 in $\mathrm{e}(G)$ span a dense subspace of ${ }^{\circ} \mathfrak{e}(G)$.

Combining this with Theorem 6 we get the following result.

Corollary. ${ }^{\circ} \mathcal{e}(G) \neq\{0\}$ if and only if rank $G=\operatorname{rank} K$.

Let $\tau$ be a unitary double representation of $K$ on a finitedimensional Hilbert space $V$. We denote by $\mathfrak{e}(G, \tau)$ the space of all $f \in \mathfrak{C}(G) \otimes V$ such that $f\left(k_{1} x k_{2}\right)=\tau\left(k_{1}\right) f(x) \tau\left(k_{2}\right)\left(k_{1}, k_{2} \in K, x \in G\right)$. Put

$$
{ }^{\circ} \mathfrak{C}(G, \tau)=\mathfrak{e}(G, \tau) \cap\left({ }^{\circ} \mathfrak{e}(G) \otimes V\right) .
$$

Theorem $10 . \operatorname{dim}^{\circ} \mathfrak{e}(G, \tau)<\infty$.

Fix a psgp $P=M A N$ of $G$. Then $M$ is a reductive group with finitely many connected components. Moreover $K_{M}=K \cap M$ is a maximal compact subgroup of $M$. Let $\tau_{M}$ denote the restriction of $\tau$ on $K_{M}$. Then it is not difficult to define the spaces $\mathrm{e}(M),{ }^{\circ} \mathrm{e}(M)$ and ${ }^{\circ} \mathcal{C}\left(M, \tau_{M}\right)$ in the same way as above.

Let $f \in \mathfrak{e}(G)$. Then we write $f^{P} \sim 0$ if

$$
\int_{M} \operatorname{conj} \phi(m) \cdot f^{P}(x m) d m=0
$$

for all $x \in G$ and $\phi \in^{\circ} \mathcal{e}(M)$. (Here $d m$ is the Haar measure on $M$ and the integral always exists.)

TheOREM 11. Let $f$ be an element in $\mathfrak{e}(G)$ such that $f^{P} \sim 0$ for all cuspidal subgroups $P$ of $G$. Then $f=0$.

Let $\mathfrak{h}_{i}(1 \leqq i \leqq r)$ be a complete set of $\theta$-stable Cartan subalgebras of $\mathfrak{g}$, no two of which are conjugate under $G$. Put $\mathfrak{a}_{\mathfrak{i}}=\mathfrak{h}_{i, R}$ and $A_{\boldsymbol{i}}=$ $\exp \mathfrak{a}_{i}$. Let $\mathfrak{C}_{i}(G)$ denote the set of all $f \in \mathfrak{C}(G)$ with the following property. 
If $P=M A N$ is any cuspidal subgroup of $G$, then $f^{P} \sim 0$ unless $A$ is conjugate to $A_{i}$ under $K$. Then $\mathfrak{e}_{i}(G)$ is a closed subspace of $\mathfrak{e}(G)$.

THEOREM 12. $\mathfrak{e}(G)=\sum_{1 \leq i \leq r} \mathfrak{e}_{i}(G)$ where the sum is direct and smooth.

Let $E_{i}$ denote the projection of $\mathfrak{e}(G)$ on $\mathfrak{e}_{i}(G)$ corresponding to the above direct sum. Then smoothness means that $E_{i}$ are continuous endomorphisms of $\mathfrak{e}(G)$. Let $\mathfrak{S}_{i}$ denote the closure of $\mathfrak{C}_{i}(G)$ in $\mathfrak{S}=L_{2}(G)$. Then $\mathfrak{S}$ is the orthogonal sum of $\mathfrak{S}_{i}(1 \leqq i \leqq r)$ and $E_{i} f$ $(f \in \mathfrak{C}(G))$ is, actually, the orthogonal projection of $f$ in $\mathfrak{S}_{i}$.

A distribution $T$ on $G$ is called tempered if it extends to a continuous linear function on $\mathfrak{e}(G)$.

Lemma 3. Let $\Theta$ be an invariant and 3-finite distribution on $G$. Then $\Theta$ is tempered if and only if we can choose $c, r \geqq 0$ such that $t^{4,5}$

$$
|D(x)|^{1 / 2}|\Theta(x)| \leqq c(1+\sigma(x))^{r}
$$

for all $x \in G^{\prime}$.

Corollary. Suppose $\Theta$ is tempered in the above lemma. Then

$$
\Theta(f)=\int_{G} f \cdot \Theta d x
$$

for $f \in \mathfrak{e}(G)$.

This follows immediately from (4) of $\S 6$. We write

$$
(\Theta, f)=\int_{G} \operatorname{conj} \Theta \cdot f d x \quad(f \in \mathfrak{e}(G)) .
$$

Let $f$ be a continuous function on $G$. We say that $f$ satisfies the weak inequality if there exist numbers $c, r \geqq 0$ such that

$$
|f(x)| \leqq c \Xi(x)(1+\sigma(x))^{r}
$$

for all $x \in G$.

Lemma 4. Let $T$ be a tempered distribution on $G$ which is both $K$ finite and 3-finite. Then $T$ satisfies the weak inequality.

Here we have to observe that a $K$-finite and $\mathbb{Z}$-finite distribution is necessarily an analytic function.

8. The projection of $\mathcal{C}(G)$ on ${ }^{\circ} \mathcal{C}(G)$. We now return to the assumptions and notation of $\S 4$. Put $B^{\prime}=B \cap G^{\prime}$ and

$$
F_{f}(b)=\Delta(b) \int_{G} f\left(x b x^{-1}\right) d x \quad\left(b \in B^{\prime}\right)
$$


for $f \in \mathfrak{e}(G)$. This integral is always convergent and $F_{f}$ is a $C^{\infty}$ function on $B^{\prime}$.

TheOREM 13. Let $f \in^{\circ} \mathrm{e}(G)$. Then $F_{f}$ extends to a $C^{\infty}$ function on $B$ and

$$
F_{f}(b)=\sum_{b^{*} \in B^{* \prime}}\left(\Theta_{b^{*}}, f\right)\left\langle b^{*}, b\right\rangle \quad(b \in B) .
$$

Now

$$
\varpi=\prod_{\alpha>0} H_{\alpha}
$$

may be regarded as a differential operator on $B$. Then, for any $f \in \mathcal{C}(G), \varpi F_{f}$ extends to a continuous function $B$.

Lemma 5. There exists a number $c_{G}>0$ such that

$$
\varpi F_{f}(1)=(-1)^{a} c_{\theta} f(1)
$$

for all $f \in \mathfrak{e}(G)$.

It is possible to determine $c_{G}$ explicitly as follows. Let be the Lie algebra of $K$. We assume, as we may, that $\mathfrak{b}$. Let $P$ be the set of all positive roots of $(\mathfrak{g}, \mathfrak{b})$ and $P_{k}$ the subset of those $\alpha \in P$ which can be regarded as roots of $(\mathfrak{l}, \mathfrak{b})$. Put

$$
\varpi_{k}=\prod_{\alpha \in P_{k}} H_{\alpha}, \quad \rho_{k}=\frac{1}{2} \sum_{\alpha \in P_{k}} \alpha
$$

and normalize the measure $d x$ as in $\S 6$.

LEMMa 6. With the above normalization of the Haar measure of $G$, we have

$$
c_{G}=[W(G / B)](2 \pi)^{q} 2^{n / 2} \varpi_{k}\left(\rho_{k}\right)
$$

where

$$
n=\operatorname{dim}(G / K)-\operatorname{rank}(G / K) .
$$

Theorem 14. For any $f \in \mathfrak{e}(G)$ and $b^{*} \in B^{*}$, define

$$
f_{b^{*}}(x)=(-1)^{q} c_{G^{-1}} \varpi\left(b^{*}\right)\left(\Theta_{b^{*}}, r(x) f\right) \quad(x \in G)
$$

where $r$ denotes the right-regular representation of $G$ on $\mathfrak{e}(G)$. Then

${ }^{6}[S]$ denotes the number of elements in a finite set $S$. 
$f_{b^{*}} \in^{\circ} \mathfrak{C}(G)$ and the series

$$
\sum_{b^{*} \in B^{*}} f_{b^{*}}
$$

converges absolutely [4(a), p. 8] in $\mathfrak{C}(G)$. Let ${ }^{\circ} f$ denote its sum. Then $f \rightarrow{ }^{\circ} f$ is a continuous projection of $\mathfrak{e}(G)$ onto ${ }^{\circ} \mathfrak{e}(G)$.

Let ${ }^{\circ} \mathfrak{S}$ denote the closure of ${ }^{\circ} \mathcal{C}(G)$ in $\mathfrak{S}=L_{2}(G)$ and $l$ the leftregular representation of $G$ on $\mathfrak{S}$.

THEOREM 15. 'S is the closure of an orthogonal sum of closed subspaces which are invariant and irreducible under l. Conversely every irreducible subspace of $\mathfrak{S}$ is contained in ${ }^{\circ} \mathfrak{S}$. Let ${ }^{\circ} \mathrm{E}$ denote the orthogonal projection of $\mathfrak{S}$ on ${ }^{\circ} \mathfrak{S}$. Then ${ }^{\circ} f={ }^{\circ} \mathrm{Ef}$ for $f \in \mathcal{C}(G)$.

9. The space $a(G, \tau)$. Fix $G$ and $\tau$ as in $\$ 2$ and $\$ 7$. We denote by $a(G, \tau)$ the space of all functions $f \in C^{\infty}(G) \otimes V$ satisfying the following three conditions:

(1) $f$ is $\tau$-spherical i.e. $f\left(k_{1} x k_{2}\right)=\tau\left(k_{1}\right) f(x) \tau\left(k_{2}\right)\left(k_{1}, k_{2} \in K, x \in G\right)$.

(2) $f$ is 3 -finite.

(3) $|f|$ satisfies the weak inequality.

For a psgp $P=M A N$ of $G$, put

$$
d_{P}(m)=\left|\operatorname{det}(A d(m))_{\mathfrak{n}}\right|^{1 / 2} \quad(m \in M A) .
$$

Let $a$ be a variable element in $A$. We say that $a \rightarrow_{P} \infty$ if $\sigma(a) \rightarrow \infty$ and there exists a number $\epsilon>0$ such that $\alpha(\log a) \geqq \epsilon \sigma(a)$ for every root $\alpha$ of $(P, A)$. Since $M A$ is a reductive group with finitely many connected components and $K_{M}=K \cap M$ is a maximal compact subgroup of $M A$, the space $\propto\left(M A, \tau_{M}\right)$ can be defined without difficulty.

TheOREM 16. For any $f \in Q(G, \tau)$, there exists a unique element $f_{P} \in Q\left(M A, \tau_{M}\right)$ such that

$$
\lim _{\substack{a \rightarrow \infty \\ P}}\left\{d_{P}(m a) f(m a)-f_{P}(m a)\right\}=0
$$

for $m \in M A$ and $a \in A$.

Given $f \in a(G, \tau)$, we write $f_{P} \sim 0$ if

$$
\int_{M}\left(\phi(m), f_{P}(m a)\right)_{V} d m=0
$$

for all $\phi \in^{\circ} \mathcal{C}\left(M, \tau_{M}\right)$ and $a \in A$. Here the scalar product is in $V$ and the integral is always convergent.

LEMMA 7. Let $f$ be an element in $a(G, \tau)$ such that $f_{P} \sim 0$ for every 
cuspidal subgroup $P$ of $G$. Then $f=0$.

Here the analogy with Theorem 11 is obvious.

For any integer $q$, let $a_{q}(G, \tau)$ denote the space of all $f \in Q(G, \tau)$ with the following property. If $P$ is a psgp of $G$, then $f_{P} \sim 0$ unless prk $P=q$.

Theorem 17. $Q_{0}(G, \tau)={ }^{\circ} \mathfrak{e}(G, \tau)$. Fix $f \neq 0$ in $Q(G, \tau)$ and choose a psgp $P=M A N$ of $G$ with the following two properties:

(1) $f_{P} \nsim 0$.

(2) $P$ is minimal with respect to condition (1).

Then for any fixed $a \in A$, the function $m \rightarrow f_{P}(m a)(m \in M)$ lies in ${ }^{\circ} \mathrm{e}\left(M, \tau_{M}\right)$.

10. The Eisenstein integral. Fix a cuspidal subgroup $P=M A N$ of $G$. Given $\psi \in \in^{\circ} \mathrm{C}\left(M, \tau_{M}\right)$, we extend it to a function on $G$ as follows:

$$
\psi(k m a n)=\tau(k) \psi(m) \quad(k \in K, m \in M, a \in A, n \in N) .
$$

For $\nu \in \mathfrak{a}_{c}{ }^{*}$ and $\psi \in \in^{\circ} \mathfrak{e}\left(M, \tau_{M}\right)$, defi ie

$$
E(P: \psi: \nu: x)=\int_{K} \psi(x k) \tau\left(k^{-1}\right) \exp \left\{\left((-1)^{1 / 2} \nu-\rho_{P}\right)\left(H_{P}(x k)\right)\right\} d k
$$

for $x \in G$. We call this the Eisenstein integral. For fixed $P$ and $\psi$, it is an analytic function of $(\nu, x)$ on $\mathfrak{a}_{c}{ }^{*} \times G$ which is holomorphic in $\nu$.

Lemma 8. $E(P: \psi: \nu) \in \mathfrak{Q}(G, \tau)$ for $\nu \in \mathfrak{a}^{*}$. If $P^{\prime}$ is another psgp of $G$, then

$$
E_{P^{\prime}}(P: \psi: \nu) \sim 0 \quad\left(\nu \in \mathfrak{a}^{*}\right)
$$

unless $P^{\prime}$ is associated to $P$.

Let $N(A)$ and $Z(A)$ respectively denote the normalizer and centralizer of $A$ in $G$. Then $Z(A)=M A$ and

$$
\mathfrak{w}=\mathfrak{w}(A)=N(A) / Z(A)
$$

is a finite group which operates on $\mathfrak{a}$ and $\mathfrak{a}^{*}$ in the usual way. Let $P^{\prime}=M A N^{\prime}$ be a psgp with the same split component $A$. Then if $\nu \in \mathfrak{a}^{*}$ is not too special (i.e. does not lie on a finite set of hyperplanes passing through the origin), there exist unique endomorphisms $c_{P^{\prime} \mid P}(s: \nu) \quad(s \in \mathfrak{w})$ of the finite-dimensional space $L={ }^{\circ} \mathfrak{C}\left(M, \tau_{M}\right)$ (Theorem 10) such that

$$
E_{P^{\prime}}(P: \psi: \nu: m a)=\sum_{s \in \mathfrak{w}}\left(c_{P^{\prime} \mid P}(s: \nu) \psi\right)(m) \exp \left((-1)^{1 / 2} s \nu(\log a)\right)
$$


for all $m \in M, a \in A$ and $\psi \in L$. Regarded as functions of $\nu, c_{P^{\prime} \mid P}(s: \nu)$ are meromorphic on $\mathfrak{a}^{*}+(-1)^{1 / 2} U$ where $U$ is an open neighborhood of zero in $\mathfrak{a}^{*}$. However it seems likely that they are actually meromorphic in the whole complex space $\mathfrak{a}_{c}{ }^{*}$. Here the analogy with Eisenstein series is quite clear (see [4(b), Chapter V] and [4(d)]).

11. The Fourier transform of a Schwartz function. From now on we shall assume for simplicity that $G$ satisfies the conditions of $\$ 4$. Let $\mathfrak{a}$ be a $\theta$-stable Cartan subalgebra of $\mathfrak{g}$ and $A$ the corresponding Cartan subgroup of $G$. Put $A_{I}=A \cap K$ and $A_{R}=\exp \mathfrak{a}_{R}$. Let $A^{*}, A_{I}{ }^{*}$ and $A_{R}{ }^{*}$ denote the character groups of $A, A_{I}$ and $A_{R}$ respectively. Then we can identify $A_{R}{ }^{*}$ with $\mathfrak{a}_{R}{ }^{*}$ and therefore $A^{*}$ with $A_{I}{ }^{*} \times \mathfrak{a}_{R}{ }^{*}$.

Let $P\left(\mathfrak{a}_{R}\right)$ denote the set of all psgps $P$ of $G$ with split component $A_{R}$. Then $P=M A_{R} N$ and $A_{I}$ is a compact Cartan subgroup of $M$. Let $3_{M}$ denote the algebra of all differential operators on $M$ which commute with left and right translations of $M$. Then for every $a^{*} \in A_{I}{ }^{*}$, we have (see $\$ 4$ ) a tempered and invariant eigendistribution $\theta_{a^{*}}$ of $\Omega_{M}$ on $M$.

Fix $a^{*} \in A^{*}$. Then $a^{*}=\left(a_{0}{ }^{*}, \nu\right)$ where $a_{0}{ }^{*} \in A_{I}{ }^{*}$ and $\nu \in \mathfrak{a}_{R}{ }^{*}$. We now define a tempered distribution $\Theta_{a^{*}}=\Theta_{a_{0}^{*}, \nu}$ on $G$ as follows:

$$
\Theta_{a^{*}}(f)=\theta_{a_{0} *}\left(g_{f, v}\right) \quad(f \in \mathfrak{C}(G)) .
$$

Here

$$
g_{f, \nu}(m)=\int_{A_{R^{X N}}} f(\operatorname{man}) \exp \left((-1)^{1 / 2} \nu+\rho\right)(\log a) d a d n, \quad(m \in M)
$$

$\rho=\rho_{P}$ and

$$
f(x)=\int_{K} f\left(k x k^{-1}\right) d k \quad(x \in G) .
$$

The Haar measures $d a$ and $d n$ are normalized as in $\$ 6$ by $d a=d \mathfrak{a}_{R}$ and $d n=d \mathfrak{n}$. One can show that $g_{f, \nu} \in \mathfrak{e}(M)$ and $\Theta_{a^{*}}$ is a tempered and invariant eigendistribution of $B$ on $G$. Moreover the definition of $\Theta_{a^{*}}$ is independent of the choice of $P$ in $\odot\left(\mathfrak{a}_{R}\right)$.

Let $\bar{A}$ be the normalizer of $A$ in $G$. Put $W(G / A)=\tilde{A} / A$. Then $W(G / A)$ is a finite group which operates in the usual way on $\mathfrak{a}$, $A$ and $A^{*}$. Let $Q$ be the set of all positive roots of $(\mathfrak{g}, \mathfrak{a})$ (under some order) and $Q_{I}$ the subset of those $\alpha \in Q$ which vanish identically on $\mathfrak{a}_{R}$. Put

$$
\varpi=\prod_{\alpha \in Q} H_{\alpha}, \quad \check{ } \mathbf{I}=\prod_{\alpha \in Q_{I}} H_{\alpha}
$$


where $H_{\alpha}$ denotes, as usual, the element in $\mathfrak{a}_{c}$ dual to $\alpha$ under the Killing form. Let $\mathfrak{a}_{I}$ be the Lie algebra of $A_{I}$ and $\lambda$ the linear function on $\mathfrak{a}_{I}$ given by

$$
\left\langle a_{0}^{*}, \exp H\right\rangle=e^{\lambda(H)} \quad\left(H \in \mathfrak{a}_{I}\right) .
$$

We define $\log a_{0} *=\lambda, \log a^{*}=\lambda+(-1)^{1 / 2} \nu$ and $\varpi_{I}\left(a^{*}\right)=\varpi_{I}\left(a_{0}^{*}\right)=\varpi_{I}(\lambda)$, $\varpi\left(a^{*}\right)=\varpi\left(a_{0}^{*}: \nu\right)=\varpi\left(\lambda+(-1)^{1 / 2} \nu\right)$. Since $\varpi_{I}^{2}$ is invariant under $W(G / A), \varpi_{I}{ }^{8}=\epsilon_{I}(s) \Phi_{I}(s \in W(G / A))$ where $\epsilon_{I}(s)= \pm 1$. Let $A_{I}{ }^{* \prime}$ denote the set of all $a_{0}{ }^{*} \in A_{I}{ }^{*}$ such that $\varpi_{I}\left(a_{0}{ }^{*}\right) \neq 0$.

Lemma 9. $\Theta_{s a^{*}}=\epsilon_{T}(s) \Theta_{a^{*}}$ for $s \in W(G / A)$ and $a^{*} \in A^{*}$.

This follows from Lemma $1(\$ 4)$.

Since $A_{I}{ }^{*}$ is a discrete group, $A^{*}=A_{I}{ }^{*} \times \mathfrak{a}_{R}{ }^{*}$ is a Lie group. By a polynomial function $p$ on $A^{*}$, we mean a function of the form

$$
p\left(a^{*}\right)=q\left(\log a^{*}\right) \quad\left(a^{*} \in A^{*}\right),
$$

where $q$ is a polynomial function on $\mathfrak{a}_{c}{ }^{*}$. Let $D\left(\mathfrak{a}_{R}{ }^{*}\right)$ denote the algebra of all translation-invariant differential operators on $\mathfrak{a}_{R}{ }^{*}$ and $D\left(A^{*}\right)$ the algebra of differential operators on $A^{*}$ generated by $\mathscr{D}\left(\mathfrak{a}_{R}{ }^{*}\right)$ and the polynomial functions on $A^{*}$. Let $\mathfrak{e}\left(A^{*}\right)$ be the space of all functions $f \in C^{\infty}\left(A^{*}\right)$ such that

$$
\mu_{D}(f)=\sup _{A^{*}}|D f|<\infty
$$

for all $D \in \mathscr{D}\left(A^{*}\right)$. We topologize $\mathfrak{e}\left(A^{*}\right)$ by means of the seminorms $\mu_{D}\left(D \in \mathscr{D}\left(A^{*}\right)\right)$. Then $C_{c}{ }^{\infty}\left(A^{*}\right)$ is dense in $\mathrm{e}\left(A^{*}\right)$. By a tempered distribution on $A^{*}$, we mean a continuous linear function on $\mathrm{e}\left(A^{*}\right)$.

For any $f \in \mathfrak{C}(G)$, put (see $\S 7$ )

$$
f_{A}\left(a^{*}\right)=\left(\Theta_{a^{*}}, f\right) \quad\left(a^{*} \in A^{*}\right) .
$$

Then $\hat{f}_{A} \in \mathfrak{C}\left(A^{*}\right)$ and $f \rightarrow \hat{f}_{A}$ is a continuous mapping of $\mathfrak{e}(G)$ into $\mathrm{e}\left(A^{*}\right)$.

Lemma 10. Let $f \in \mathfrak{e}(G)$. Then

$$
\dot{f}_{A}\left(s a^{*}\right)=\epsilon_{I}(s) f_{A}\left(a^{*}\right) \quad\left(s \in W(G / A), a^{*} \in A^{*}\right) .
$$

This is an immediate consequence of Lemma 9.

Let $A^{*}(S)$ denote the set of all points $a^{*} \in A^{*}$ where $\varpi\left(a^{*}\right)=0$.

Now fix a complete set $\mathfrak{a}_{i}(1 \leqq i \leqq r)$ of $\theta$-stable Cartan subalgebras of $\mathfrak{g}$, no two of which are conjugate under $G$. Let $A_{i}$ denote the corresponding Cartan subgroup. Put $\hat{f}_{i}=\hat{f}_{A_{i}}(f \in \mathfrak{C}(G))$ and define $\epsilon_{i, I}(s)$ $\left(s \in W\left(G / A_{i}\right)\right)$ as above. 
THEOREM 18. Suppose we are given, for each $i(1 \leqq i \leqq r)$, a tempered distribution $T_{i}$ on $A_{i}{ }^{*}$ such that: ${ }^{7}$

(1) $T_{i}^{s}=\epsilon_{i, I}(s) T_{i}\left(s \in W\left(G / A_{i}\right)\right)$,

(2) $\sum_{1 \leq i \leq r} T_{i}\left(\hat{f}_{i}\right)=0$ for all $f \in \mathfrak{C}(G)$.

Then ${ }^{8}$ Supp $T_{i} \subset A_{i}{ }^{*}(S)(1 \leqq i \leqq r)$.

We think of $\left(\hat{f}_{i}\right)_{1 \leqq i \leqq r}$ as the Fourier transform of $f \in \mathbb{e}(G)$.

12. The Plancherel formula for $G$. Define $\mathfrak{b}, B, W=W(\mathfrak{g} / \mathfrak{b})$ and $\varpi$ as in $\$ 4$. (We assume that $B \subset K$.) For any $f \in \mathfrak{C}(G)$, put

$$
{ }^{*} F_{f}(b)=[W]^{-1} \sum_{s \in W} \varpi F_{f}\left(b^{s}\right) \quad(b \in B) .
$$

Then (see $\S 8$ ) $* F_{f}$ is a continuous function on $B$. We now use the notation of Theorem 18. Let $d_{i} a^{*}$ denote the Haar measure on $A_{i}{ }^{*}$.

THEOREM 19. There exist unique continuous functions $C_{i}$ on $A_{i}{ }^{*} \times B$ $(1 \leqq i \leqq r)$ with the following properties:

(1) $C_{i}\left(a^{*}: b\right)=0$ if $\varpi_{i, I}\left(a^{*}\right)=0\left(a^{*} \in A_{i}{ }^{*}, b \in B\right)$.

(2) $C_{i}\left(s a^{*}: b\right)=\epsilon_{i, I}(s) C_{i}\left(a^{*}: b\right)\left(s \in W\left(G / A_{i}\right)\right)$.

(3) We can choose numbers $c, p \geqq 0$ such that ${ }^{9}$

$$
\left|C_{i}\left(a^{*}: b\right)\right| \leqq c\left(1+\left\|\log a^{*}\right\|\right)^{p}
$$

for all $a^{*} \in A_{i}^{*}$ and $b \in B$.

$$
{ }^{*} F_{f}(b)=\sum_{1 \leq i \leq r} \int_{A_{i}^{*}} C_{i}\left(a^{*}: b\right) \hat{f}_{i}\left(a^{*}\right) d_{i} a^{*}
$$

for $f \in \mathfrak{e}(G)$ and $b \in B$.

The uniqueness is an immediate consequence of Theorem 18.

Combining this with Lemma 5, we get the following result.

Corollary. $\quad(-1)^{q} c_{G} f(1)=\sum_{1 \leq i \leq r} \int_{A i^{*}} C_{i}\left(a^{*}: 1\right) \hat{f}_{i}\left(a^{*}\right) d_{i} a^{*}$ for $f \in \mathfrak{C}(G)$.

This is substantially the Plancherel formula for $G$.

The proof of Theorem 19 is based on a long induction on the rank of $G$. It makes essential use of the differential equations connecting the functions $F_{f}$ and $F_{z f}(z \in \mathcal{B}, f \in \mathcal{C}(G))$. The basic idea of utilizing these differential equations to obtain a theorem of the above type,

${ }^{7} W\left(G / A_{i}\right)$ operates on the space of distributions on $A_{i} *$ in the obvious way.

${ }^{8}$ Supp $T_{i}$ denotes the support of $T_{i}$.

${ }^{9}$ The Euclidean norm on $g$ (see $\S 6$ ) defines the structure of a Hilbert space on $g_{e}$ and hence also on $\mathfrak{a}_{b}$ and $\mathfrak{a}_{c}{ }^{*}$ for $\mathfrak{a}=\mathfrak{a}_{i}$. 
goes back to R. P. Langlands who had worked out the case of $\mathrm{SL}(2, \boldsymbol{R})$ by this method in 1962 .

Fix $i(1 \leqq i \leqq q), a_{0}^{*} \in A_{i, I}^{*}$ and put $\mathfrak{F}_{i}=\mathfrak{a}_{i, R}^{*}$. Then the function

$$
C_{i}\left(a_{0}^{*}: \nu: b\right) \quad\left(\nu \in \mathfrak{F}_{i}, b \in B\right)
$$

is of class $C^{\infty}$ on $\mathfrak{F}_{i} \times B^{\prime}$ and it is analytic in $\nu$. In fact it is a rather simple function which, for a fixed $b \in B^{\prime}$, is meromorphic on $\mathfrak{F}_{i, c}$.

13. The relation between the Plancherel measure and the asymptotic behaviour of certain Eisenstein integrals. Fix $i(1 \leqq i \leqq r)$ and drop it from the notation. Now put

$$
q\left(a_{0}^{*}: \nu\right)=C_{i}\left(a_{0}^{*}: \nu: 1\right) \quad\left(a_{0} \in A_{I}^{* \prime}, \nu \in \mathfrak{F}\right) .
$$

We wish to obtain a formula for $q\left(a_{0}{ }^{*}: \nu\right)$.

For any $\nu \in \mathfrak{F}_{c}$, put $\nu=\nu_{R}+(-1)^{1 / 2} \nu_{I}\left(\nu_{R}, \nu_{I} \in \mathfrak{F}\right)$. Fix $P=M A_{R} N$ in $P\left(\mathfrak{a}_{R}\right)$ and for any $\delta>0$ let $\mathfrak{F}_{c}(\delta)$ denote the set of all $\nu \in \mathfrak{F}_{c}$ such that $\left\|\nu_{I}\right\|<\delta$. Then if $\delta$ is sufficiently small, we have defined (see $\$ 10$ ) the meromorphic function $c_{P \mid P}(1: \nu)$ on $\mathfrak{F}_{c}(\delta)$ with values in the space of endomorphisms of ${ }^{\circ} \mathfrak{C}\left(M, \tau_{M}\right)$. Let $\alpha_{1}, \cdots, \alpha_{l}$ be all the distinct simple roots of $\left(P, A_{R}\right)$. Define $H_{j} \in a_{R}$ by $\alpha_{i}\left(H_{j}\right)=\delta_{i j}(1 \leqq i, j \leqq l)$. Let $\mathfrak{F}_{c}(P)$ denote the set of all $\nu \in \mathfrak{F}_{c}$ such that $\nu_{I}\left(H_{j}\right)<0 \quad(1 \leqq j \leqq l)$. Then $c_{P \mid P}(1: \nu)$ is actually holomorphic on $\mathfrak{\Im}_{c}(P)$. Put $\bar{N}=\theta(N)$. Given $x \in G$, we can write it uniquely in the form $x=k \operatorname{man}$ ( $k \in K$, $m \in M, a \in A, n \in N)$ where $\theta(m)=m^{-1}$. Define $\kappa(x)=k$ and $\mu(x)=m$.

Lemma 11. Put $\rho=\rho_{P}$ and $H(x)=H_{P}(x)(x \in G)$. Then

$$
\begin{aligned}
\left(c_{P \backslash P}(1: \nu) \psi\right) & (m) \\
& =\int_{\bar{N}} \psi\left(m \mu(\bar{n})^{-1}\right) \tau(\kappa(\bar{n}))^{-1} \exp \left[-\left((-1)^{1 / 2} \nu+\rho\right)(H(\bar{n}))\right] d_{0} \bar{n}
\end{aligned}
$$

for $\psi \in \in^{\circ} \mathfrak{C}\left(M, \tau_{M}\right), \nu \in \mathfrak{F}_{c}(P)$ and $m \in M$. Here the integral is convergent and the Haar measure $d_{0} \bar{n}$ on $\bar{N}$ is so normalized that

$$
\int_{\bar{N}} e^{-2 \rho(H(\bar{n}))} d_{0} \bar{n}=1 .
$$

Let $V_{0}$ be the subspace of all $v_{0} \in V$ such that $\tau(k) v_{0}=v_{0} \tau(k)$ for all $k \in K$. Fix $a_{0}{ }^{*} \in A_{I}^{* \prime}, v_{0} \in V_{0}$ and define $\psi\left(a_{0}{ }^{*}: v_{0}\right)=\psi \in \in^{\circ} \mathcal{C}\left(M, \tau_{M}\right)$ by

$$
\psi(m)=\int_{K_{M}} \theta_{a_{0}^{*}}\left(m k^{-1}\right) \tau(k) v_{0} d_{0} k \quad(m \in M),
$$


where $d_{0} k$ is the normalized Haar measure on $K_{M}$ and $\theta_{a_{0}}^{*}$ has the same meaning as in $\$ 11$. Let $\mathfrak{F}^{\prime}\left(a_{0}{ }^{*}\right)$ denote the set of all $\nu \in \mathfrak{F}$ such that $\varpi\left(a_{0}^{*}: \nu\right) \neq 0$. Then $c_{P \mid P}(1: \nu) \psi$ is holomorphic in $\nu$ for $\nu \in \mathfrak{F}^{\prime}\left(a_{0}^{*}\right)$. Put

$$
\|\phi\|_{M}^{2}=\int_{M}|\phi(m)|^{2} d m \quad\left(\phi \in{ }^{\circ} \mathfrak{C}\left(M, \tau_{M}\right)\right),
$$

$q_{G}=\frac{1}{2} \operatorname{dim} G / K$ and $q_{M}=\frac{1}{2} \operatorname{dim} M / K_{M}$.

THEOREM 20. There exists a constant $c_{0}(P)>0$ (independent of $a_{0}{ }^{*}$ and $\tau$ ) such that

$$
c_{0}(P) q\left(a_{0}^{*}: \nu\right)\left\|c_{P \mid P}(1: \nu) \psi\right\|_{M}^{2}=(-1)^{q} G^{+q} \varpi_{I}\left(a_{0}^{*}\right)\|\psi\|_{M}^{2}
$$

for $\nu \in F^{\prime}\left(a_{0}{ }^{*}\right)$ and $\psi=\psi\left(a_{0}^{*}: v_{0}\right)\left(v_{0} \in V_{0}\right)$.

Actually it is possible to determine $c_{0}(P)$ explicitly. Moreover it is not difficult to show that for any given $a_{0}{ }^{*} \in A_{I}^{* \prime}$, we can choose $\tau$ in such a way that $\psi\left(a_{0}^{*}: v_{0}\right) \neq 0$ for some $v_{0} \in V_{0}$.

14. The p-adic case. First we recall some standard facts about algebraic groups [1].

Let $\Omega$ be a field. By an $\Omega$-group, we mean a (linear) algebraic group defined over $\Omega$. Let $G$ be a connected and reductive $\Omega$-group. By a parabolic subgroup $P$ of $G$, we mean an algebraic subgroup which contains a Borel subgroup of $G$. We say that $P$ is $\Omega$-parabolic if it is parabolic and defined over $\Omega$. Let $N$ denote the (unipotent) radical of $P$. Then $N$ is an $\Omega$-subgroup and $P$ is the normalizer of $N$ in $G$.

Now assume that $\Omega$ is a $\mathfrak{p}$-adic field (i.e. a locally compact field with a discrete valuation). Let $G$ denote the set of all $\Omega$-rational points of $G$. Then $G \subset \mathrm{GL}(n, \Omega)$ for a suitable $n \geqq 1$. GL $(n, \Omega)$, being an open subset of a vector space over $\Omega$ of dimension $n^{2}$, is a locally compact group. Since $G$ is closed in $\mathrm{GL}(n, \Omega)$, it is also locally compact. Moreover it is easy to verify that it is separable and unimodular.

By a parabolic subgroup $P$ of $G$, we mean a subgroup of the form $P=G \cap P$, where $P$ is an $\Omega$-parabolic subgroup of $G$. Then $P$ is uniquely determined by $P$. Put $N=G \cap N$ where $N$ is the radical of $P$. Let $C_{c}(G)$ denote the space of all continuous complex-valued functions on $G$ with compact support. For any $f \in C_{c}(G)$, define

$$
f^{P}(x)=\int_{N} f(x n) d n \quad(x \in G),
$$

where $d n$ is the Haar measure of the unimodular group $N . f$ is said 
to be a cusp form if $f^{P}=0$ for all parabolic subgroups $P \neq G$. Let ${ }^{\circ} C_{c}(G)$ denote the space of all cusp forms in $C_{c}(G)$.

Let $\pi$ be an irreducible unitary representation of $G$ and $\mathfrak{S}_{\pi}$ the representation space of $\pi$. We say that $\pi$ is supercuspidal if there exist nonzero elements $\phi, \psi$ in $\mathfrak{S}_{\pi}$ such that the function $x \rightarrow(\phi, \pi(x) \psi)$ $(x \in G)$ lies in ${ }^{\circ} C_{c}(G)$. Let ${ }^{\circ} \mathcal{E}(G)$ denote the set of all classes $\omega \in \mathcal{E}(G)$, which contain supercuspidal representations. It is obvious that ${ }^{\circ} \mathcal{E}(G) \subset \mathcal{E}_{2}(G)$.

Let $G_{0}$ be an open subset of $G$ and $C_{c}^{\infty}\left(G_{0}\right)$ the space of all locally constant complex-valued functions on $G_{0}$ with compact support. By a distribution $T$ on $G_{0}$, we mean a linear mapping of $C_{c}^{\infty}\left(G_{0}\right)$ into $C$. Let $F$ be a function on $G_{0}$ which is locally summable with respect to the Haar measure $d x$ of $G$. Then we say that $T=F$ if

$$
T(f)=\int_{G} F \cdot f d x
$$

for all $f \in C_{c}^{\infty}\left(G_{0}\right)$.

THEOREM 21. Let $\pi$ be a square-integrable representation of $G$. Then for any $f \in C_{c}^{\infty}(G)$, the operator

is of the trace class.

$$
\pi(f)=\int_{G} f(x) \pi(x) d x
$$

Since every neighborhood of 1 in $G$, contains an open compact subgroup, this is an immediate consequence of the corollary of Theorem 5.

Define

$$
\Theta_{\pi}(f)=\operatorname{tr} \pi(f) \quad\left(f \in C_{c}^{\infty}(G)\right) .
$$

Then $\Theta_{\pi}$ is a distribution on $G$ which depends only on the class $\omega$ of $\pi$. Hence we may denote it by $\Theta_{\omega}$. Then $\Theta_{\omega}$ is invariant and the mapping $\omega \rightarrow \Theta_{\omega}\left(\omega \in \mathcal{E}_{2}(G)\right)$ is injective.

TheOREM 22. Let $\mathfrak{S}_{\pi}$ denote the representation space of a squareintegrable representation $\pi$ of $G$. Fix $\phi, \psi \in \mathfrak{S}_{\pi}$. Then

$$
\begin{aligned}
(\phi, \psi) \Theta_{\pi}(f) & =d(\pi) \int_{G}\left(\phi, \pi(x) \pi(f) \pi\left(x^{-1}\right) \psi\right) d x \\
& =d(\pi) \int_{G} d x \int_{G} f(y)\left(\phi, \pi\left(x y x^{-1}\right) \psi\right) d y
\end{aligned}
$$

for $f \in C_{0}{ }^{\infty}(G)$. 
This is an easy consequence of the Schur orthogonality relations.

The function $D$ and the regular set $G^{\prime}$ are defined in exactly the same way as in $\S 2$. Then $D$ is a regular function on $G$ and $G^{\prime}$ is an open and dense subset of $G$ whose complement is of measure zero.

TheOREM 23. Fix $\omega \in^{\circ} \mathcal{E}(G)$. Then there exists a locally constant function $F$ on $G^{\prime}$ such that $\Theta_{\omega}=F$ on $G^{\prime}$.

It seems very likely that $F$ is actually locally summable on $G$ and $\Theta_{\omega}=F$. In any case, the analogy with the results of $\S 2$ is obvious.

For any $\alpha \in \Omega$, we define its absolute value $|\alpha|$ in the usual way so that

$$
d(\alpha t)=|\alpha| d t
$$

for any additive Haar measure $d t$ on $\Omega$.

LEMma 12. If $\Omega$ has characteristic zero, the function $|D|^{-1 / 2}$ is locally summable on $G$.

Put $\Phi=|D|^{1 / 2} F$ in the notation of Theorem 23. It seems reasonable to expect that $\Phi$ remains bounded on $G^{\prime}$.

By a Cartan subgroup $A$ of $G$, we mean a subgroup of the form $A=G \cap A$ where $A$ is a maximal $\Omega$-torus in $G$. Let $\Phi_{A}$ denote the restriction of $\Phi$ on $A^{\prime}=A \cap G^{\prime}$.

Lemma 13. There exists a compact subset $C$ of $A$ such that $\Phi_{A}=0$ outside $C$. Moreover if $A$ is compact and $\Omega$ has characteristic zero, then $\Phi_{A}$ remains bounded on $A^{\prime}$.

When $G=\operatorname{SL}(2)$ and the characteristic of the residue field of $\Omega$ is not 2, the characters of $G$ have been explicitly computed by Sally and Shalika [8] and their results agree with the above statements.

15. Two problems. Let me conclude these lectures by discussing two problems (or conjectures) in the $p$-adic case.

Fix an open compact subgroup $K_{0}$ of $G$ and let ${ }^{\circ} C_{c}\left(G / / K_{0}\right)$ denote the space of all functions in ${ }^{\circ} C_{c}(G)$ which are constant on double cosets of $K_{0}$. Then one would like to show that, in case prk $G=0$,

$$
\operatorname{dim}{ }^{\circ} C_{c}\left(G / / K_{0}\right)<\infty .
$$

Here the analogy with Theorem 10 is obvious.

In view of Theorem 5 , it would be enough to prove that

$$
\inf _{\omega \in \in^{\circ} \varepsilon(G)} d(\omega)>0 .
$$

Actually I am inclined to believe that the numbers $d(\omega)\left(\omega \in \mathcal{E}_{2}(G)\right)$ are 
all integers under a suitable normalization of the Haar measure of $G$.

$K$ being any open compact subgroup of $G$, an affirmative answer to the above question would imply that

$$
\sup _{\omega \in \varepsilon(G)}[\omega: \oslash]<\infty
$$

for $\downarrow \in \mathcal{E}(K)$, provided $\Omega$ has characteristic zero.

In order to state the second problem, we have to recall a recent theorem of Bruhat and Tits. Let $\left(P_{0}, A_{0}\right)$ be a mincuspidal pair in $G$ and $P_{0}=M_{0} N_{0}$ the corresponding Levi decomposition [4(c), §2]. Put $A_{0}=G \cap A_{0}$ and for any root $\alpha$ of $\left(P_{0}, A_{0}\right)$, let $\xi_{\alpha}$ denote the corresponding character of $\boldsymbol{A}_{0}$. Let $A_{0}^{+}$be the set of all points $a \in A_{0}$ where $\left|\xi_{\alpha}(a)\right| \geqq 1$ for every root $\alpha$ of $\left(P_{0}, A_{0}\right)$. Define $\mathfrak{w}\left(A_{0}\right)$ as in $[4(\mathrm{c}), \S 2]$.

Theorem (Bruhat-Tits). We can choose an open and compact subgroup $K$ of $G$ with the following properties:

(1) $G=K P_{0}$.

(2) $G=K A_{0}^{+} C K$, where $C$ is a finite subset of $M_{0}$.

(3) Every element of $\mathfrak{w}\left(\boldsymbol{A}_{0}\right)$ has a representative in $K$.

(4) If $(P, \boldsymbol{A}) \succ\left(P_{0}, \boldsymbol{A}_{0}\right)$ is a cuspidal pair and $P=M N$ the corresponding Levi decomposition, then $P \cap K=(M \cap K)(N \cap K)$.

(5) Put $K_{M}=K \cap M$ and ${ }^{*} P_{0}=M \cap P_{0}$ in the notation of (4). Then if we replace $\left(G, P_{0}, \boldsymbol{A}_{0}, K\right)$ by $\left(M,{ }^{*} P_{0}, \boldsymbol{A}_{0}, K_{M}\right)$, the above four conditions are again fulfilled.

We keep to the notation of the above theorem. Let $\tau$ be a unitary representation of $K$ on a finite-dimensional Hilbert space $V$ and let $C_{c}(G, \tau)$ denote the space of all functions $f$ from $G$ to $\operatorname{End}(V)$ with compact support such that

$$
f\left(k_{1} x k_{2}\right)=\tau\left(k_{1}\right) f(x) \tau\left(k_{2}\right) \quad\left(k_{1}, k_{2} \in K, x \in G\right) .
$$

Then $C_{c}(G, \tau)$ is an algebra under convolution. On the other hand, let $V_{P}$ be the subspace of all $v \in V$ such that $\tau(n) v=v$ for all $n \in N \cap K$. Let $E_{P}$ denote the orthogonal projection of $V$ on $V_{P}$. Put

$$
\tau_{M}(m)=\tau(m) E_{\boldsymbol{P}} \quad\left(m \in K_{M}\right) .
$$

Then $\tau_{M}$ may be regarded as a representation of $K_{M}$ on $V_{P}$ and so we can consider the algebra $C_{c}\left(M, \tau_{M}\right)$.

Let $d_{r} p$ denote a right-invariant Haar measure on $P$ and define the function $\delta_{P}$ on $P$ by the relation

$$
d_{r}(q p)=\delta_{P}(q) d_{r} p \quad(q \in P) .
$$


For any $f \in C_{c}(G, \tau)$, put

$$
f^{(P)}(m)=\delta_{P}(m)^{1 / 2} \int_{N} f(m n) E_{P} d n \quad(m \in M),
$$

where $d n$ is a Haar measure on $N$. Then $f^{(P)} \in C_{c}\left(M, \tau_{M}\right)$ and the mapping $\mu_{P}: f \rightarrow f^{(P)}$ is actually a homomorphism of $C_{c}(G, \tau)$ into $C_{c}\left(M, \tau_{M}\right)$. We would now like to assert that $C_{c}\left(M, \tau_{M}\right)$ is a finite right-module over $\mu_{P}\left(C_{c}(G, \tau)\right)$. If this is true, much of the theory for real groups [4(a), Part II] can be imitated in the p-adic case.

\section{REFERENCES}

1. A. Borel and J. Tits, Groupes rèductifs, Inst. Hautes Etudes Sci. Publ. Math No. 27 (1965), 55-150. MR 34 \#7527.

2. I. M. Gelfand, Automorphic functions and the theory of representations, Proc. Internat. Congress Math. (1962) Inst. Mittag-Leffler, Djursholm, 1963, pp. 74-85. MR $28 \# 1$.

3. R. Godement, Sur les relations d'orthogonalité de V. Bargmann. I: Démonstration générale, C. R. Acad. Sci. Paris 225 (1947), 657-659. MR 9, 134.

4. Harish-Chandra, (a) Discrete series for semisimple Lie groups. II, Acta Math. 116 (1966), 1-111. MR 36 \#2745. (b) Automorphic forms on semisimple Lie groups, Lecture notes in Math., no. 62, Springer-Verlag, Berlin and New York, 1968. MR 38 \#1216. (c) Eisenstein series over finite fields, Proc. Stone Jubilee Conf., Chicago, 1968. (d) Some applications of the Schwartz space of a semisimple Lie group, Lecture Series, Modern Anal. and Appl., George Washington Univ., 1968.

5. G. W. Mackey, Infinite-dimensional group representations, Bull. Amer. Math. Soc. 69 (1963), 628-686. MR 27 \#3745.

6. C. C. Moore, Decomposition of unitary representations defined by discrete subgroups of nilpotent groups, Ann. of Math. (2) 82 (1965), 146-182. MR 31 \#5928.

7. L. Pukánszky, Leçons sur les représentations des groupes, Monographies de la Société Mathématique de France, no. 2, Dunod, Paris, 1967. MR 36 \#311.

8. P. J. Sally, Jr, and J. A. Shalika, Characters of the discrete series of representations of SL(2) over a local field, Proc. Nat. Acad. Sci. U.S.A. 61 (1968), 1231-1237.

9. L. Schwartz, Théorie des distributions. Tome I, Actualités Sci. Indust., no. 1091, Hermann, Paris, 1950. MR 12, 31.

Institute for Advanced Study, Princeton, New Jersey 08540 